about. With regard to the complications just enumerated, they are of very little importance, being extremely rare; and it is very unlikely, should the complication be diseased structure, whether malignant or otherwise, that it should be confined entirely to the tissues in front of the hernial sac, and not extend to the cellular tissue or structures on either side; should it, on the other hand, be a tumour, cyst, or parasitic growth, it would certainly be beneficial to the patient to be relieved of such complication, and that such disease or abnormal growth should be allowed to escape, or suppurate away by a direct, rather than by an indirect opening; that the operation should relieve the patient of his strangulated hernia and his abnormal growths at one and the same time, rather than by cutting on one side, relieve the hernia and defer the growths for some future proceedings. It is very true that erysipelas, suppuration, and even gangrene sometimes attack the wound made in the usual operation, and also that some patients labouring under disease of vital organs, have died after operations for the relief of strangulated hernia, but surely it is not meant to be asserted that ossification of the aorta, disease of the brain, erysipelas, suppuration or gangrene resulted from the sac having been opened; this result, or mischief, depending as it must have done upon upon some constitutional idiocyncrasy, would have equally followed any wound inflicted on the patient, whether according to Gay's, Luke's, or the usual method. Neither is bleeding into the abdomen necessarily fatal; I have seen two cases of wounded artery in the opera. tion for femoral hernia. In both the vessels were carefully secured, and the patients did very well.

Lawrence* relates a case in which the epigastric artery was completely divided without occasioning any hæmorrhage during the operation, or previously to the patient's death, as was proved by post-mortem examination; also an instance of femoral hernia, where, after the stricture was divided, the wound immediately filled with arterial blood; the mouth of the vessel could not be distinguished, but the patient fainted, and the bleeding ceased. The patient ultimately recovered.

As to the risk of hamorrhage in the operations for hernia he entirely agrees with Breschet, who quotes the opinions of Lallemant and Richerand, that the fear of bleeding in these operations had been much exaggerated.

Velpeau also relates a case, where, in the body of a person who had died from a wound of the abdomen, he found the epigastric artery had been completely divided; there had been comparatively little bleeding, which had ceased spontaneously. $t$

Harley-street, July, 1849.

\section{THE NECK AS A MEDICAL REGION; HIDDEN SEIZURES; PAROXYSMAL AFFECTIONS OF THE NERVOUS SYSTEM.}

\section{By MARSHALL HALL, M.D., F.R.S., \&c.}

(SIXTH SERIES.)

$I$ now beg to resume what $I$ have said in my former papers on this important subject, observing only, that the present must be considered partly as supplementary to, and partly as recapitulatory of, those papers, which, in their turn, contain some facts which may not be found in the present one. They must be read together.

I take this opportunity of thanking several correspondents for their communications, and of requesting the continued aid of my professional brethren in this investigation.

\section{Introductory.}

We have all witnessed the dire effects of paroxysm of the nervous system-stupor, delirium, amentia, paralysis, syncope, \&c. We have also all witnessed these, and other affections of this kind, arising from other causes. I believe I state for the first time, that a great number of these latter cases have, in reality, arisen from paroxysmal seizures unobserved, nndetected, and therefore unsuspected; in a word, from what I would designate hidden seizures.

In this manner, apoplexy, paralysis even, mania, amentia, \&c., have taken their origin, and they are then, as much as any other affections of the nervous system, paroxysmal in their form, and, happily, sometimes more transient, or, at least, less lasting than the same affections from other causes, generally organic lesion.

I have known hemiplegia and mania, from this source, to cease in a day or a few days. Nay, $I$ have known mere par-

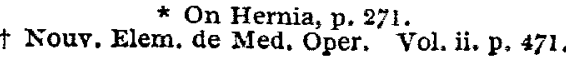

tial paralysis and delirium to cease in a few hours, or even more promptly still.

I have known the case to be considered as arachnitis or effusion, or softening of the brain, in cases in which a speedy recovery has plaiuly demonstrated that a change, more of a functional character, and less of that of organic lesion, had happily been the source of the symptoms; that the disease, however it had assumed an apoplectic, paralytic, maniacal, perhaps syncopal form, had not involved any of those organic changes which too frequently exist in apoplexy, in paralysis, in mania, and in syncope. How important the diagnosis! I have often indeed witnessed cases which have been involved. in the deepest mystery, until the occurrence of a previous paroxysm, or previous paroxysms, unknown to any oneperhaps occurring in the night, or from home-had been de. tected.

The idea of hidden paroxysms is new in pathology, and it is one of the utmost moment for the diagnosis, prognosis, and treatment.

And now the question arises,-What is the nature,-what are the causes, their mode of action, their effects, their varied sequence and character, in these cases of hidden seizure?

I believe this question to have remained untraced, nay, absolutely unthought of before; and it is at once as recondite and as momentous a question as any in pathology. In one case, the effect of emotion, I observed the eye, the features, and the head, all drawn to the right side, with stupor, hemiplegia, followed by delirium, and speedy recovery. In another, the effect of an indigestible meal, there was a similar distortion of the neck, starting of the external jugular, extreme turgescence of the eyes and eyelids, stupor, amentia, of a less transient character. In a third case, the patient, a female, was talking incessantly, the platysma myoides and the external jugular, on each side, being raised in rigid and prominent relief. In other cases, the cleido-mastoid, the omo-hyoid, the trapezius, the subclavius, and other muscles of the Neck are contracted on the other veins, constituting what $I$ have ventured to designate trachelismus* and phlebismus, $\neq$ or more emphatically, sphagiasmus $\neq$ or rhachiasmus,§ according as the jugular or the vertebrate are chiefly compressed.

From this compression of the veins, their roots or origins, and the methæmatous vessels, or methæmata,ll and, in their turn, the arteries themselves, are distended. The last seem to throb, and the whole are in an erectile condition.

The cerebrum, the medulla oblongata, and the medulla spinalis, are in a proportionate state of irritation or compression, and of proportionately augmented or diminished function

This altered function displays itself in the varied symptoms of paroxysmal nervous affections, to which $I$ have briefly adverted, and which occur and subside as the erectile condition of the vessels occurs and subsides, the attack being generally: sudden, the subsidence slow.

Such are the links of this deeply interesting chain of causes and effects:-

1. Excitement-1, emotion; 2, gastric; 3, enteric; 4, uterine irritation.

2. Sleep, with its sphagiasmus.

3. Contracted muscles of the Neck; trachelismus.

4. Compressed veins of the neck, phlebismus, - sphagiasmus, rhachiasmus.

5. An erectile condition of the veins, methæmatous vessels, and arteries.

6. Irritation or congestion of the nervous centres.

7. Proportionate excitement or diminution of their function, erethismus, or catalysis.

8. Variation of the symptoms with that of the nervous centre chiefly affected, and of the degree of congestion, and of irritation or of catalysis.

9. Slow and gradual, and perhaps imperfect, subsidence of these effects of the seizures, with, perhaps,

10. Augmented susceptibility to their return.

In addition to this recapitulation, I may repeat-

1. That the seizure may remain unobserved, and therefore hidden.

2. That its effects may be regarded as an original, and not. be recognised as a secondary and paroxysmal disease.

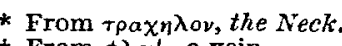

$$
\begin{aligned}
& + \text { From } \phi \lambda \in \psi \text {, vein. }
\end{aligned}
$$

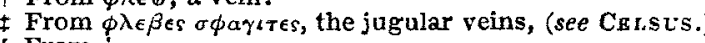$$
\text { From } \rho a x 45
$$

So I have designated the blood-channels which exist intermediately between the minute and capillary branches of the arteries and roots of the reins, which I first discovered, and described, in 1831, and in which all the changes induced in and by the blood in the pneumonic and systemic circulations are effected, the veins, heart, and arteries being mere machinery to effect and conduct its movement. 
3. That the diagnosis is of the utmost moment to the prognosis and the treatment.

Having thus given a brief sketch of my subject, I propose to resume each topic embraced by it, and treat it more at large in the following pages.

\section{The Causes.}

When we obscrve the obvious effort of emotion on the "Anatomy of Expression," in the countenance, especially the eye and the mouth; in the neck, especially on affecting the platysina myoides, the cleido-mastoid; in the hands, in the extremities, in the attitude, in the respiration, the speech, \&c., we cease to be surprised that its influence should reach the omo-hyoid, the trapezius, \&c., and so affect the deeply-seated muscles of the neck.

When we observe the influence of gastric, enteric, and uterine or hysteric irritation in inducing the severer or severest forms of paroxysmal affection of the nervous system, we cease to be surprised at minor effects of these irritations, and at the hidden forms of seizure or paroxysm, which arise from them, with their varied and varying effects, mysterious until their paroxysmal nature and origin are detected.

Another cause of this kind of seizure or paroxysm is sleep; and another, the sudden interruption of sleep.

The influence of mind, or of attention on the muscular system, has not yet been fully traced. We see it in the eyelid; we observe it in respiration; we are struck with the absence of mind in the countenance, and in a thousand ways. The muscular system is continually under the influence of volition, of thought, of emotion, of spinal action or tone. The first three of them are gently withdrawn during sleep. The eyelid droops, the eyeboll is turned upwards and inwards; the levator-palpebra being less under the influence of tone than the orbicularis, and the oblique, than the other muscles of the eye. The pupil contracts by the action of the circular fibres of the iris. The muscles of the larynx and palate are less equipoised by volition, and the respiration becomes less perfect, and audible. Every one is aware how different is the aching and restless position of the body when we are awake, from the quiet observed under the influence of " tired Nature's sweet restorer," when all is tone.

Should we wonder that the muscles of the Neck partake of the condition of the rest of the muscular system; that they should contract gently, like the orbicularis, as volition and emotion are withdrawn from fatigue and languor; and that, as a consequence of this contraction, compression of the jugular and other veins occurs-and sleep. Sleep, then, is a sort of slight apoplexy already. It differs from apoplexy in degree, and in being physiological. In apoplexy all the phenomena of sleep exist in an augmented form; the respiration becomes still more audible and more stertorous, and irregular and imperfect. The pupil of the eye, instead of being contracted, is dilated.

But the condition during sleep may be aggravated, and then one of those seizures of which I am about to treat may supervene; there may be a cerebral or a spinal seizure or paroxysm! The actual seizure is only a difference in degree from the physiological condition.

Let emotion be added-let a turbulent dream supervene -and then we have a second cause adding its influence to that of sleep, and a cerebral or spinal seizure may occur, a frequent form of this being night-mare; or the patient may awake, having lost the power of the hand, or having bitten the tongue? In other instances there is restlessness, or headach, or vertigo, perhaps "sick headach," which may or may not continue the next dày.

Dr. - - during life, could never indulge in a sound sleep in the night, or in a sleep during the day, without suffering from vertigo.

But the jar or shock to the nervous system by the sudden disturbance of the sleep, and the effect of later or of earlier hours than usual, or of interruption of the sleep, not of a sudden or violent kind; anything unusual, in a word, may also issue in a paroxysmal seizure. One medical gentleman, liable to slight and transierit cerebral seizures, is sure to be affected the day after being disturbed in the night. One young lady had a seizure after having been at the opera; another, in carriage, after having been called earlier than usual to take a journey.

It is under the head of the causes of paroxysmal affection of the nervous system, that $I$ ought to notice the subject of "sick-headach." Sick-headach is the type of a Class of these affections, combining nausea or sickness with some other symptom or symptoms, as headach, vertigo, momentary loss of conciousness, perhaps "falling."
Many years ago, I described a peculiar malady, which combined nausea, vomiting, falling, perhaps spasmodic affection, a cold damp perspiration, \&c.

The attack of apoplexy is sometimes preceded or accompanied by sickness; so it is represented by Hogarth.

This sickness, this condition of the stomach, may, however, be cause or effect. The affection with which it is associated may be ranked amongst the most trifling or the most dire of human maladies.

The whole presents a subject for investigation to the scientific physician, of the most intense interest, and one of those which will ever distinguish him from the low routinier, or empiric. And yet I have known more than one patient so afflicted, and so surrounded by danger to life, mind, or limb, trusted, in the ignorant confidence of wife or parent, to globules, of which they knew nothing, for a malady of which they knew less. It is the medical superstition with its ignorance, and is scarcely exceeded by the hideous and benighted idolworship of the Hindoos. Thus recently perished poor Lady Blessington.

3. The Muscles of the Neck; Trachelismus.

There is not in medicine a subject more strangely overlooked than the condition of the muscles of the Neck; as they are submitted to actual observation.

The action of the platysma myoides, of the cleido-mastoid, of the trapezius, may be seen; that of other muscles of the neck, more deeply seated in its anterior, and in its posterior and lateral regions, may be felt. I repeat, then, that I appeal to observation.

That these muscles should contract, forcibly contract, from the influence of volition, without inducing violent and injurious compression of the subjacent veins, is one of the most marvellous events of physiology, and, doubtless, arises from their nearly balanced antagonism. But let the condition of these muscles be induced, not by volition, but by emotion or by reflex action, even within the boundaries of physiology; or let it be pathological in kind and in degree, and then we observe, or may observe, its effects on the subjacent or adjacent venous structures.

Dr. Todd, who is so lavish of his feeble and futile criticisms on my labours, says that he has seen the platysma myoides contract without inducing distention of the external jugular. So have $\mathrm{I}$, a thousand times. The platysma myoides is a very broad muscle, as its name imports; and it is not the contraction of any part of this muscle, as of the ample anterior portion, which can compress the external jugular vein, but of its posterior and inferior fourth part. It is of this part expressly that I would be understood to speak, when stating that the action of the platysmanyoides influences the flow of blood entering the external jugular. I have seen the fibres of the platysma, and the course of this vein, "stand out" in equal and most prominent "relief," in numerous cases; as I have seen the fibres of the anterior front of the platysma become prominent, without distention of the vein.

Besides, in further speaking of the platysma myoides, I have, to obviate silly criticism, added, "and other muscles of the neck," in more than one instance. In effect, the platysma rarely, perhaps never, acts alone. And even in compression of the external jugular with the action of the posterior inferior portion of the platysma, that of the subclavian and that of the trapezius may be, and doubtless usually is, conjoined. The whole subject is one for cautious observation, experiment, investigation, in a word, and not for hasty aud worthless criticism.

Within twelve hours of writing these lines, I have witnessed a case of severe convulsive attack with Mr. Burke, of Upper Montagu-street. The platysma, the cleido-mastoid, the trapezius, the muscles of the anterior and posterior and lateral portions of the neck, were rigid as so many cords or ropes, with violent torti-collis and other distortions, and I did not observe the external jugular to be distended. In a case already mentioned, on the other hand, the neck remained straight, while both platysmas and both external jugulars were equally prominent. The candid anatomist will best understand why in one case the external, in another the internal, jugular, and in a third the vertebral vein, shall bo chiefly or, it may be, solely affected.

I wonder that the celebrated author of "The Anatomy of Expression" did not investigate this subject. It would have been full of interest to him.

Thus, "contraction of the posterior inferior portion of the platysma myoides, and of other muscles of the neck," is, under the influence of emotion, the cause of blushing, a state of congestion of the external venous and methrmatous channels, otten diffused over the cheeks, forehead, neck, and bosom. 
Thus, contraction of various muscles, especially the cleidomastoid, the omo-hyoid, \&c., is the cause of congestion of the internal jugulars, and of its dire consequences, whilst the contraction of the muscles in the posterior region of the neck compresses the vertebrals.

These actions are usually various, combined so as to induce the varied appearances and symptoms observed in patients affected with paroxysms. With them, morbid conditions of the larynx and of the respiratory apparatus are also conjoined.

Let us not begin so very soon to carp and criticise, but let us devote ourselves to the search after truth, and so honour our profession and benefit mankind.

\section{The Veins of the Neck-Phlebismus.}

Compression of the veins of the neck induces the condition of the venous roots, of the intermediate methæmatous channels, and in their turn, of the arteries, observed in erectile tissues under the similar condition of the circulating system.

The idea of tendency of blood to a part is obviously an error. There is no power, no director of power, which can produce any such effect.

But let a vein, or rather, let the veins be compressed and obstructed, and then it is easy to conceive the full effect on the arrière circulation. The circulation in the neck and head is impeded, the vessels are turgid and congested, and, possibly even ruptnred, and in the case of the neck, the delicate tissue of the nervous centres is variously irritated or compressed, erethized, or catalyzed.

The veins compressed may be the more decply-seated veins, as the internal jugular, or the vertebral, and be "out of sight." But the effects of impeded venous circulation are observed in the symptoms, - in the suffused condition of the eye and eyelid, and in the tension and throbbing of the temporal artery and of the carotid.

But the veins compressed may be the more superficial veins, and not the external jugular only, but the other superficial veins of the neck, the facial vein, \&c., and then the face is suffused, the nervous centres being, comparatively, spared. In some instances, ecchymosed spots are seen occupying the eyelids or adjacent parts of the face.

In one case the convulsive affection involved the muscles of the face, the platysma, the cleido-mastoid, the trapezins, and even the muscles of the left arm, leaving partial paralysis of the under lip and arm, and the tongue and lip were bitten; and yet the insensibility was not constantly complete, nor did any mental imbecility remain.

There is no variety in this phlebismus, as in the trachelismus, and the symptoms, which may not occur. The suffision or congestion may be superficial, or, with the symptoms, cerebral, or spinal. The suffusion is obvious, and it may be viewed as denoting the state of things in the more deeply seated congestion.

A still clearer idea of this condition may be obtained by observing the circulation in the web of the frog, assisted by a ligature lightly applied to the thigh; the flow of blood becomes retarded, pulsatory, oscillatory, "congested," in the minute veins and arteries, and in the methæmatous vessels, with repletion, distention, and perhaps ecchymosis, and the arteries are seen to throb.

\section{Hidden Seizures.}

We may be summoned to witness a case of convulsive disease, and when the violence of the paroxysm is over, we see its effects on the cerebral system, these effects being variously stupor, hemiplegia, paralysis, mania, amentia, \&c.

In other cases we are called to witness various shades of stupor, of paralysis, of delirium, of loss of mind, but not a word, or a hint, or a suspicion, occurs of any previous seizure. We conclude that there is cerebral disease, inflammation, softening, \&c.; but we are shortly surprised to find that our patient recovers more speedily than we had anticipated and prognosticated. We are congratulating ourselves on this favourable result, when the prospect is again clonded by a return of the symptoms, perhaps assuming a different form; still no convulsive affection has been observed, no suspicion of any such paroxysm arisen!

Such a paroxysm may, however, have occurred and have been hidden, that is, unobserved. This may take place in two ways. In the first, the patient may have been attacked in the night, or away from home and from his friends, and an attack, obvions enough in itself, may have passed unwitnessed or unrecorded.

In the second, the convulsion may have been limited to the deeply-seated muscles, and to the deeply-seated reins of the neck, and have been actually hidden even from near observers, the patient, as stated by Heberden-“ ad se redeat prius quam ab adstantibus animadvertatur."

Such are the events which I would designate as hidden seizures, and to which I beg earnestly to draw the attention of the profession. In ALI cerebral affections, but especially the SEDDEN and the REPEATED, we should carefully inquire for evidence of a seizure or seizures hitherto hidden!

In one most interesting case, I was called to a patient affected with the mildest form of mania. There was merely an erroneons idea abont his affairs, and a degree of suspicion. The symptoms subsided; he appeared recovering. A return of symptoms took place, and now there was, for several weeks, violent mania. The patient again recovered, but was again doomed to be disappointed; he became affected with a sort of amentia, and we suspected effusion. This idea was rendered untenable by a third speedy amendment. The patient was again worse; and now I made the most minute inquiries for some sign of paroxysmal seizure. I found that, in walking in the drawing-room, he had experienced a "shudder," but I could obtain no more explicit information on this interesting and important point. At length, a fourth relapse took place, in the form of a distinct convulsive seizure, followed by transient paralysis of the lips and of the arm, and still greater amentia than before. This seizure was followed by another, and this by another.

The case had plainly been one of hidden seizure!

And thus a flood of light is thrown upon one of the most obscure of obscure diseases! With this light a ray of hope is also associated, for, difficult of cure as they may be, I need scarcely observe, that a paroxysmal seizure inducing conges. tion is less incurable than organic disease of the nervous centres. Even paralysis of this character is frequently transitory, and never hopeless.

In this view there is, however, at least one exception. It is that in which the excitant of the seizure is intra-cranial or intra-spinal. Thus a tumour, affections of the arachnoid, any disease within the cranium or spine, may become the exciting cause of paroxysms.

6. New View in Pathology.

The action of certain causes of disease of the nervous centres is immediate on the nervous centres themselves, as that of many physical or chemical agents, such as a blow, a coup de soleil, \&c.

Other causes act through the incident nerves, as in traumatic tetanus.

Others again act through the blood, as in hydrophobia.

But in the case before us there is a sort of double or changed mode of action. Certain excitants, mental or physical, induce contraction of the muscles of the neck, (trachelismus,) and this action compresses the veins, (inducing phlebismus,) and in this methodic course the nervous centres suffer. Then is induced congestion, and then recovery, more or less prompt or tardy, perfect or imperfect.

This congestion may obtain chiefly in the tissues of the face, or in the cerebrum, or in the medulla oblongata or spinalis, and in their associated functions, according as one or other of the muscles of the Neck may be chiefly affected. It is usually, I believe, the hand or side that is convulsed which is afterwards found to be enfeebled or paralyzed.

\section{The Diagnosis.}

The effects of seizures, and especially of hidden seizuresthat is, congestion and its effects-must be distinguished from all lesions of the nervous centres of a physical kind.

The diagnosis is established by observing-

1. The suddenness of the attack;

2. The comparatively speedy recovery from it;

3. Its recurrence

4. Its varied effects, organic disease being marked by a more gradual and unitorm progress.

We must especially inquire for evidence of hidden seizures. This evidence is sometimes supplied by a servant, sometimes by a bitten tongue or lip; or there may be minute ecchymoses on the temple, the forehead, or the eyelid, or suffusion of the conjunctiva.

A seizure may be suspected in cases in which the patient has been subjected to emotion or agitation of mind, or has partaken of an indigestible meal, or has suffered from constipation, or on the recurrence of the catamenia.

Flatus, sickness, the slightest spasmodic affection, in the beginning especially, are also diagnostic. I have already mentioned "sick headach" as the type of paroxysmal affections.

The paroxysmal character of this Class of diseases, even of paralysis itself, is a point in the diagnosis to which I would earnestly call the attention of the profession. 


\section{Dissection; Anatomy and Physiology.}

In order to prepare ourselves for the observation of paroxysmal affections of the nervous centres, we ought most carefully to dissect the $N e c k$, its muscles and its veins, and having variously injected the external jugulars, the internal jugulars, the vertebrals, in various ways, we should trace their course in the intra-cranial and intra-spinal cavities respectively.

We shall thus be best enabled to judge of the effect of different seizures according to the special muscles and veins chiefly involved in them. We shall thus be enabled to explain the suffused forehead, the brief oblivions, the slight spasmodic movements, in the various milder forms of seizure and the same appearances aggravated in their more aggravated forms, the effects of compressed external jugulars, internal jugulars, or vertebrals, respectively.

We ought also to become familiar with the precise intracranial and intra-spinal distribution of the veins especially. It is very peculiar. It must influence the effect of extra-cranial and extra-spinal compression. And it must be thoroughly understood before the physician is prepared to investigate the subject pathologically and practically.

I propose to pursue this piece of anatomy.

I wish especially to show that the scientific physician, as well as the scientific surgeon, must be an anatomist.

\section{Observation; Experiments.}

It is most interesting to observe, in the various cases of seizure which come under our notice, the order in which the muscles of the neck seem to contract, and the effects on the venous and nervous systems.

In one case the platysma myoides was seen on both sides in active contraction, and in prominent relief, and the external jugulars distended, the patient talking incessantly, the head being erect and undrawn to either side.

In another case the head was first drawn forwards, and then to one side, by the action, and then by the unequal action of the cleido-mastoids. There was very transient unconsciousness only.

In a third the more deeply seated muscles were violently contracted with stupor, followed by delirium, and transitory paralysis of one side.

In a fcurth, the larynx is closed, and the tongue protruded and bitten, not the cerebrum only, but the medulla oblongata being irritated.

In varions cases, transient unconsciousness, syncopal pallor, perhaps " falling," are observed.

Then come other forms of seizure, of the most formidable kind-convulsion, coma, mania, death; or partial loss of the power of mind, or of limb, or of speech, or of a special sense, \& c.

The anatomist and the physiologist alone can trace these effects to their causes, can undertand them, can become the scientific observer and physician in such circumstances.

Besides observation, I believe experiment may be called to our aid in this inquiry.

What would be the effect on dogs, for example, of cansing contraction in the various muscles of the neek by means of the electro-dynamic apparatus? Could we induce coma or convulsion in this manner?

What would be the effect of ligature on one or more of the veins, in various order and combination?

Having thus induced congestion and its effects, and continued them for a time, what are appropriate remedies?

And here I would also ask, may not the influence of chloroform be employed during the painful, often the merely preparatory, part of such experiments? and, might not observation, however painful, at the abattoir, yield us important facts?

\section{The Treatment.}

The treatment comprises the prevention and the mitigation of the paroxysins, and of their effects.

The causes, psychical and physical, must be avoided.

The susceptibility of the nervous system must be subdued if possible, by every tonic means, and especially by pedestrian or equestrian exercisis, early hours, the sponge showerbath, \&c.

The prima vixe and the secretions should be kept in order by daily antacid eccoprotic aperients.

And, especially, the system should, $I$ think, be kept under the long-sustained light mercurial influence. From this influence, if conjoined with exercise in the open air, no harm whatever results; and, from sume extraordinary cases, I am perfectly satisfied that it does intinite good-mitigating the number and force of the attacks, and in due time-that is, a very long time -subduing their effects. In one case, after twenty-five severe attacks, with severe hemiplegia, the patient has been well for five years-so well as to use his paralyzed hand as an engraver. But, then, he has never been quite free from the effect of mercurial remedies, and if he has attempted to omit them, ho has had no equivocal warning of the continued necessity for them. Another patient left three months ago for Jersey, continued there in as much quiet as possible, taking the mild aperients and mercurials, and is returned, marvellously re stored to firmness of mind. It was a case of hidden seizure induced by emotion, and followed by depression of the mental powers, with tendency to tears, \&c.

It appears to me that, in addition to these measures, frequently cupping in the neck near the occiput, crossing the incisions, and taking but little blood at each operation, is the most powerful means of relieving the state of venous congestion which constitutes the third link in the chain of cause and effects in this class of maladies.

It requires much decision of purpose, and great enthusiasm, to carry out the just treatment in these trying cases. If all is not accomplished, it is apt to be concluded that nothing is accomplished. Though the seizures be mitigated in number and in degree, in a given space of time, yet if they do not cease, they and their effects entirely - an impossible event, reminding us of the saying of Johnson-the physician is supposed to have assumed a responsibility of which he never dreamt, and his office is a thankless one. Mry young readers should fortify themselves against this kind of injustice, and persevere, notwithstanding, in well-doing.

Manchester-square, July, 1849.

\section{REMARIKS ON THE CHOLERA, AS IT EXISTS IN HOLLAND.}

Bx WILLIAM ALEXANDER, M.D.,

PHYSICIAN TO THE HALIFAX INFIHMARY AND DISPENSARY.

Having just returned from an excursion tour to the Netherlands, and a visit to the University of Leyden, some opportunities have been afforded me of observing the epidemic cholera, as it now prevails thronghont Holland, which, if detailed, may not be without interest to some of your readers.

To those well acquainted with the physical features of this interesting land of dykes, and the insuperable difficulties of adequate drainage, but little surprise will be felt that Holland when visited by the choleraic atmospheric influence, should suffer very severely. The canals, which in the towns intersect and everywhere run parallel with the streets, are stagnant pools, which have received the drainage of centuries. At Amsterdam, the effuvium, in warm, dry weather, from these gigantic sewers, is beyond any power of mine to describe; and the Hague, Haarlem, and some parts of Leyden, appeared the only agreeable cities in Western Holland.

The cholera, during the last two months, has been epidemic in the Brielle, Rotterdam, and Amsterdam. At the latter place, which numbers a population of ncarly a quarter of a million, $\mathrm{I}$ saw, on July 3rd, in the Almshouse in the Kloveniers Burgheval, upwards of sixty patients in the various stages of this intractable disease. To the courtesy and intelligence of Drs. Van Deventer and Brevet $I$ am indebted for much of the information $I$ gleaned on the subject, and to these gentlemen has been entrusted the charge of this hospital at Amsterdam, the only one hitherto appropriated to the reception of cholera cases in that city.

The general aspect of the disease was precisely identical with that I had previously seen in India, in 1828, and at Whitby, in 1833; as elsewhere, filthy localities, and persons whose health is already impaired, furuish the chief victims. The general belief among the profession seems to be in a modified contagion, as to its propagation under a gentral, indefinable constitution of the atmosphere. Drs. Derenter and Brevet think with me, that sanitary arramements are the only rational means whereby we can hope effectively to check the origin and spread of epidemic disease of evcry kind.

The means of prevention, seeing that all modes of cure fail to restore more, at best, than fifty per cent. of those cases which have passed into the algide stage, must, in all countries, in future, be the great desideratum to be sought for-a task of no small difficulty in Holland, a great part of which has no natural outlet for its drainage.

A diarrhoea of pale-coloured evacuations has usually, though not invariably, been found, on inquiry, to precede the ricewater stage. At Amsterdam, the treatment pursued, with about the usual average success in the algide crisis, consists in the application of artificial heat, and the internal administra. 